\title{
Research on Characteristics of Electrostatic Wear-Site and Oil- Line Sensor with Theoretical and Comprehensive Analysis
}

\author{
L. I. U. Ruochen $\mathbb{D}^{1,2}$ B. E. I. Shaoyi, ${ }^{1}$ G. U. Meng $(\mathbb{D})^{1}$ W. A. N. G. Han $\mathbb{D}^{2,3}$ \\ and S. U. N. Jianzhong $\mathbb{1}^{3}$ \\ ${ }^{1}$ School of Automobile and Traffic Engineering, Jiangsu University of Technology, Changzhou 213001, China \\ ${ }^{2}$ Center for Advanced Life Cycle Engineering (CALCE), University of Maryland, College Park, MD 20742, USA \\ ${ }^{3}$ College of Civil Aviation, Nanjing University of Aeronautics and Astronautics, Nanjing 211106, China
}

Correspondence should be addressed to L. I. U. Ruochen; liuruochen_nuaa@163.com

Received 5 November 2021; Revised 24 January 2022; Accepted 3 February 2022; Published 26 February 2022

Academic Editor: Agustin Herrera-May

Copyright (@) 2022 L. I. U. Ruochen et al. This is an open access article distributed under the Creative Commons Attribution License, which permits unrestricted use, distribution, and reproduction in any medium, provided the original work is properly cited.

\begin{abstract}
As a unique monitoring technique, electrostatic monitoring has been proved to be effective on prognostics and health management of tribological system by using electrostatic sensors. Wear-site sensor (WSS) with a flat circle probe and oil-line sensor (OLS) with a ring probe are two typical sensor types for electrostatic monitoring. However, this developing technique has not been applied in actual industry manufacture so far due to the united characteristics on electrostatic sensors. This paper first presents the mathematical models of both WSS and OLS for theoretical simulation. Then a series of contrast analysis on sensor characteristics between WSS and OLS with varied sensor structure parameters are presented. The results show that the distributions of spatial sensitivity for WSS or OLS itself only are similar, while they are quite different between WSS and OLS. Both WSS and OLS are symmetrical and their distributions can be simplified in one phase for further investigations. The theoretical efficiency curves have close relationship between WSS and OLS. The investigations of sensor performances on characteristics and a presented unified three level system have offered a better electrostatic sensor application in future.
\end{abstract}

\section{Introduction}

On-line condition monitoring with smart sensors is an essential part in the prognostics and health management (PHM) of mechanical equipment [1-3]. The performance of sensors used in the system have a great impact on the effectiveness of monitoring [4-8]. Nowadays, different from a unique developing technique which is called the electrostatic monitoring method has been investigated based on electrostatic charging phenomena $[9,10]$. This technique has been proved to be effective for tribological contacts by using electrostatic sensors [11-14].

Electrostatic monitoring was firstly invented and installed to monitor the deterioration of engine components with turbine engine monitoring systems in aircraft [15-17]. Then, it was further developed from gas path to the tribo-contracts [18-21]. After a series of experimental works on sliding rolling and bearing wear systems, it has been demonstrated that this novel technique has considerable advantages in condition monitoring of tribological systems with its high sensitivity and resolution [22-32]. Unlike monitoring the traditional secondary effects such as temperature and vibration, electrostatic sensing technique is considered as a direct measurement for friction and wear. It can provide early warnings before severe failures for essential maintenance. Wear-site sensor (WSS) and oilline sensor (OLS) are two typical sensor types in electrostatic monitoring systems [21]. WSS detects charges on the worn surfaces while OLS detects charges in lubricant circulations.

Although many researches have been conducted on the electrostatic monitoring technique with experiments, it is still challenging with limited efforts taken in the structures and performance of electrostatic sensors. The characteristics and parameters of electrostatic sensors should be clarified prior to the detection. However, only a few researches have 
been carried out and focused on sensors in electrostatic monitoring. Harvey [22] used a method to detect charges with an oil droplet rig, while Morris [11] proposed the unique sensor efficiency along with its field of view for electrostatic sensors by the detection of multiple charged steel balls. Liu $[26,27]$ introduced a mathematical model for OLS and further established a model for WSS based on charging principles with analysis through both simulation and experiments. Mao [26-29] developed a new debris recognition method for OLS and verified in a gearbox test.

Through previous studies, we can find them no further investigations into the parameters and performance on both WSS and OLS based on physical model have been conducted. The exact relationship of characteristics between WSS and OLS are not confirmed. If we want to apply the two sensors to actual industrial production, the lack of a unified system for all electrostatic sensors has limited the optimization of design and better applications. So the specific mathematical models for both WSS and OLS using the same electrostatic sensing principle have been individually introduced follow their own structure. The performance of electrostatic sensors on the extracted significant parameter spatial sensitivity is analysed with its distribution in 3D spatial simulation. A series of contrast between wear-site sensors (WSSs) and oil-line sensors (OLSs) have been investigated based on their characteristics. A unified three level system for characteristics of electrostatic sensors, processed to be suitable for both WSSs and OLSs, has finally been presented.

\section{Knowledge of Electrostatic Monitoring}

2.1. Structure of Electrostatic Sensor. The basic features of typical used electrostatic WSS and OLS in electrostatic monitoring system are listed in Table 1, along with their structures as shown in Figure 1. The common of the two type electrostatic sensors is that they all consist of three basic layers, i.e. a sensing layer, an insulation layer and a shielding layer, from inside to outside [20]. The sensing layer is the inner part to generate the inducted charge with a conductive probe surface when inducing charges are detected in the region, while the surface shape of WSS is flat and OLS is ring. The insulation layer is the middle part to protect the generated charge in probe with insulation materials. The shielding layer is the outer part to shield the electrostatic signal from electromagnetic interference. Both the electrostatic WSS and OLS have signal output connectors corresponding to their probe (normally WSS has only one and OLS has two) and installation parts as well.

The located position of these two types electrostatic sensors are different. WSSs are normally positioned close to the surface of wear components, e.g. sliding, rolling, and bearing surface, to detect charges generated around the area on the process of operating. OLSs are normally positioned in the oil line of lubrication system together with tube or pipe, which is far away from the critical components, only to detect the charged debris. It is obvious that these tribological systems without oil line can only apply WSSs for electrostatic morning and need to save enough space around the wear site for sensor installation. In other tribological systems, OLS need to be installed before filters and have the same inner diameter with the pipe in the oil line to avoid interfering the lubricating system.

2.2. Principle of Electrostatic Sensing. Electrostatic monitoring technique is a complex process from the initial charge generation in tribological system to the final acquisition and analysis of electrostatic signals, as seen in Figure 2. Based on the previous investigations, five causes (i.e. tribo-charging, tribo-emission, tribo-electrification, surface charging and debris charging) were thought to be the possible charge generation mechanisms in tribo-contacts $[8,20]$. When an inducing charging source (e.g. charged debris or lubricant charge species) passes the front of the sensor probe, an opposite charge response can then be detected by its usual inductive processes. Considering that multiple charge sources is the combination of single charge source, only single source is discussed in this investigation to simplify the analysis. It is worth mentioning that an OLS commonly has two identical probes with two outputs, seen in Figure 1, while a WSS has only one probe with one output. The two ring probes of the OLS would not interfere each other with a relatively far distance and their corresponding outputs are nearly the same. Hence the model of OLS is modified as one ring probe to simplify the analysis and consist with WSS.

\section{Mathematical Model of Electrostatic Sensor}

3.1. Sensor Space Coordinate System. According to the previous investigations [14-17], the first step to build electrostatic sensor model is to define the space coordinate system. Considering both WSS (flat circular sensing surface) and OLS (ring shape sensing surface) have a good space symmetry, it is easy to define the origin of the space rectangular coordinate system, as shown in Figure 3. The location of origin, i.e. point $\mathrm{O}$, for WSS is the sensing surface centre with $\mathrm{X}$ and $\mathrm{Y}$ axis also in this plane; while for OLS is the ring probe geometrical centre with $\mathrm{X}$ and $\mathrm{Y}$ axis in radial direction. The $\mathrm{Z}$ axis is vertical to the XOY plane which is the axial direction for both WSS and OLS.

In order to differentiate the two electrostatic sensors and their coordinate systems, the subscript is added with "WS" to represent WSS and "OL" to represent OLS in the whole investigations below. The most important parameter for electrostatic WSS is the probe diameter $D_{\mathrm{WS}}$, which equals to $2 R_{\mathrm{WS}}$ (the radius of the sensing surface) and is shown in Figure 3(a), while the probe length in axial direction and insulation layer thickness in radial direction are ignored. However, unlike WSS, the electrostatic OLS probe has two important parameters. One is the diameter of the ring probe $D_{\mathrm{OL}}$, which equals to $2 R_{\mathrm{OL}}$ (the radius of the ring probe). Another is the length of the ring probe in axial direction $H_{\mathrm{OL}}$ and equals to $2 L_{\mathrm{OL}}$. Both the two parameters are shown in Figure $3(\mathrm{~b})$, while the probe the thickness in radial direction is ignored. The formulations and calculations below are all based on the built space coordinate systems and set probe parameters.

3.2. Model of Electrostatic WSS and OLS. For WSS, assuming the coordinate of inducing point charge $q_{\mathrm{WS}}$ is $\mathrm{A}\left(x_{\mathrm{WS}}, 0, z_{\mathrm{WS}}\right)$, i.e. within $\mathrm{X}_{\mathrm{OZ}}$ plane. A selected small surface $d s_{\mathrm{WS}}$ is $\mathrm{A}^{\prime}$ at 
TABLE 1: Features of the two types of electrostatic sensors.

\begin{tabular}{lcc}
\hline Sensor features & WSS & OLS \\
\hline Basic structure & Three layers (sensing layer, insulation layer and shielding layer) \\
$\begin{array}{l}\text { Sensing surface shape } \\
\text { Relative position to the critical components }\end{array}$ & Flat & Ring \\
Most limit factor & Close to & Far away (in the oil line) \\
\hline
\end{tabular}

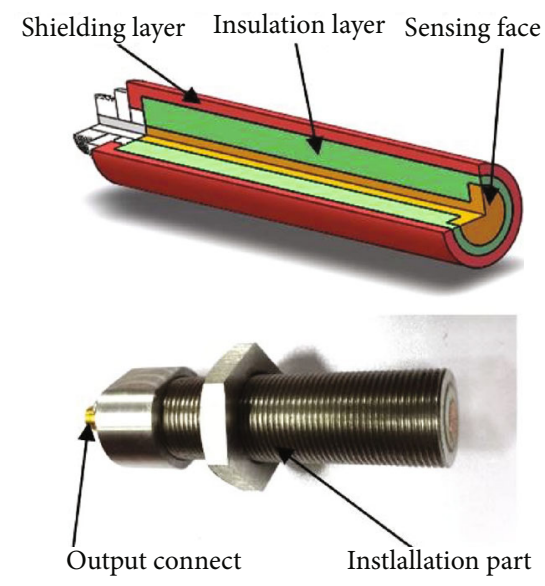

(a) WSS
Sensing rings Shielding layer Insulation layer
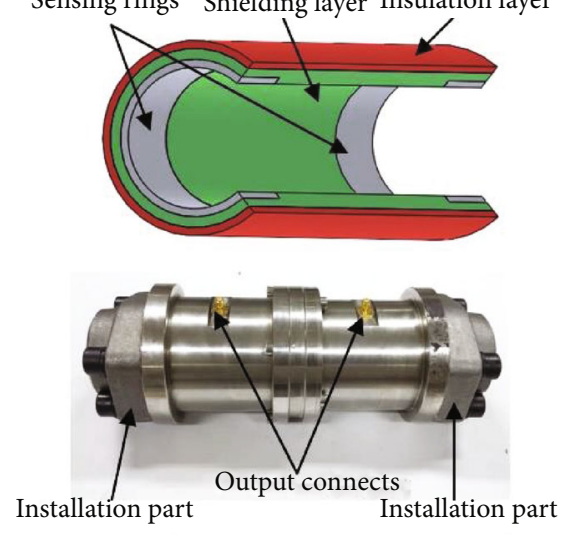

(b) OLS

FIgURE 1: The schematic diagram and picture of electrostatic sensor basic structure.

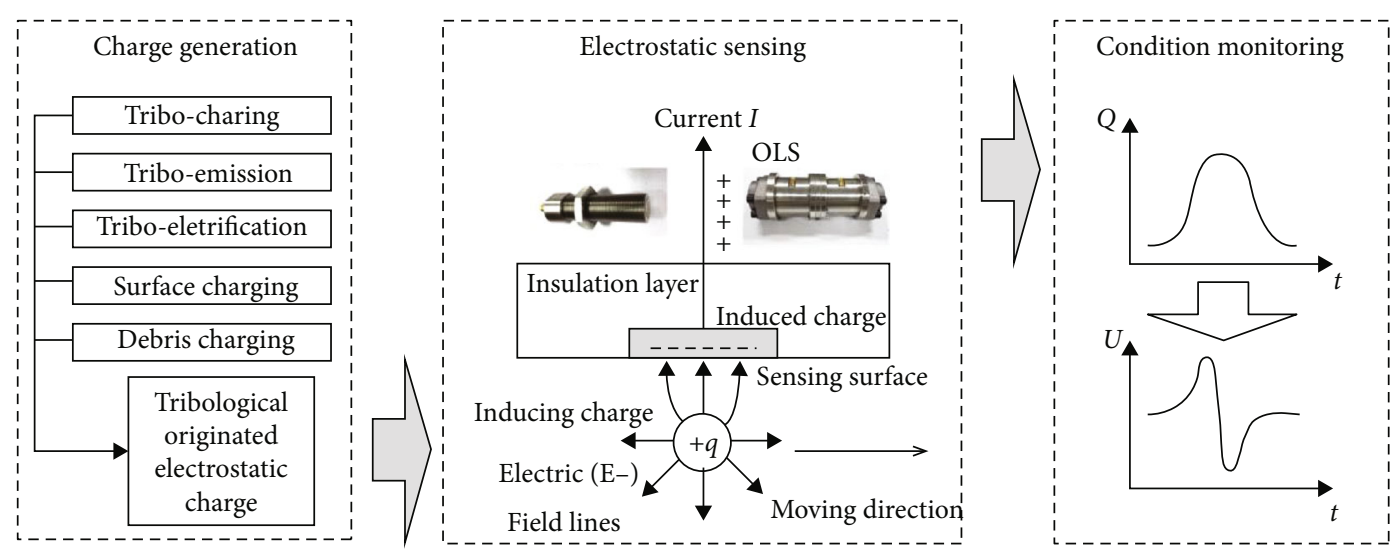

FIGURE 2: A schematic diagram of electrostatic monitoring principle.

the sensing surface has a distance $r_{\mathrm{WS}}$ to the origin. $\varphi_{\mathrm{WS}}$ is angle between $\mathrm{OA}^{\prime}$ and $\mathrm{X}$ axis (i.e. the projection of inducing charge at $\mathrm{X}_{\mathrm{OY}}$ plane to the origin). Then according to the reference [26], the probe induced charge $\mathrm{Q}_{\mathrm{WS}}$ can be calculated through Coulomb's law with Gauss theorem to get the modified following equation:

$$
Q_{w s}\left(x_{w s}, Z_{w s}\right)=-\frac{q_{w s} Z_{w s}}{4 \pi} \int_{0}^{R_{w s}} \int_{0}^{2 \pi} \frac{r_{w s}}{\left(Z_{w s}^{2}+r_{w s}^{2}+x_{w s}^{2}-2 r_{w s} x_{w s} \cos \varphi_{w s}\right)} d \varphi_{w s} d r_{w s}
$$

The sensor characteristics for WSS then can be simulated through equation (1) with four parameters, i.e. $q_{\mathrm{wS}}$, $R_{\mathrm{WS}}, x_{\mathrm{WS}}$ and $z_{\mathrm{WS}}$. It is obvious that the built WSS model is suitable for all the locations of various space point change, which can be equivalent transformed into XOZ plane owing to its symmetrical shape.

Just the same as WSS, for OLS, the assuming coordinate of inducing point charge $q_{\mathrm{OL}}$ is $B\left(x_{\mathrm{OL}}, 0, z_{\mathrm{OL}}\right)$, i.e. within $\mathrm{XOZ}$ plane. A small selected surface $d s_{\mathrm{OL}}$ (e.g. at point $B^{\prime}$ ) was selected at XOY plane. $\varphi_{\mathrm{OL}}$ is the angle between $\mathrm{O} B^{\prime}$ and $\mathrm{X}$ axis (i.e. the projection of inducing charge to the origin $\mathrm{O}$ ). 


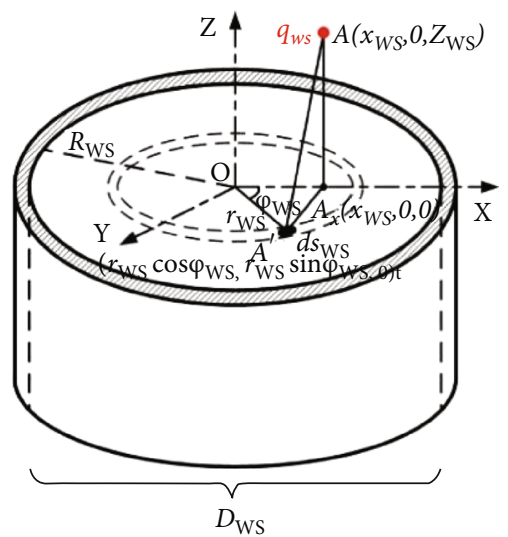

(a) WSS

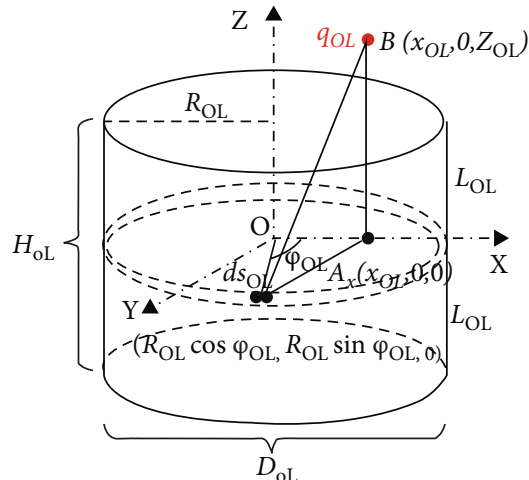

(b) OLS

FIGURE 3: The coordinate system of electrostatic sensor with an inducing point charge.

Then according to the reference [27], the probe induced charge $Q_{\mathrm{OL}}$ can be calculated through Coulomb's law with Gauss theorem to get its modified following equation:

$$
Q_{\mathrm{OL}}\left(x_{\mathrm{OL}}, z_{\mathrm{OL}}\right)=\frac{R_{\mathrm{OL}} q_{\mathrm{OL}}}{2 \pi} \int_{0}^{\pi} \frac{R_{\mathrm{OL}}-x_{\mathrm{OL}} \cos \varphi_{\mathrm{OL}}}{{R_{\mathrm{OL}}}^{2}+x_{\mathrm{OL}}{ }^{2}-2 R_{\mathrm{OL}} x_{\mathrm{OL}} \cos \varphi_{\mathrm{OL}}}\left(\frac{z_{\mathrm{OL}}+L_{\mathrm{OL}}}{\sqrt{\left(z_{\mathrm{OL}}+L_{\mathrm{OL}}\right)^{2}+\left(R_{\mathrm{OL}}{ }^{2}+x_{\mathrm{OL}}{ }^{2}-2 R_{\mathrm{OL}} x_{\mathrm{OL}} \cos \varphi_{\mathrm{OL}}\right)}}-\frac{z_{\mathrm{OL}}-L_{\mathrm{OL}}}{\sqrt{\left(z_{\mathrm{OL}}-L_{\mathrm{OL}}\right)^{2}+\left(R_{\mathrm{OL}}{ }^{2}+x_{\mathrm{OL}}{ }^{2}-2 R_{\mathrm{OL}} x_{\mathrm{OL}} \cos \varphi_{\mathrm{OL}}\right)}}\right) d \varphi_{\mathrm{OL}}
$$

Similarly, the sensor characteristics for OLS can be simulated through equation with five parameters, i.e. $q_{\mathrm{OL}}, R_{\mathrm{OL}}$, $L_{\mathrm{OL}}, x_{\mathrm{OL}}$ and $z_{\mathrm{OL}}$. It is obvious also that the built OLS model is suitable for all the locations of various space point change, which can be equivalent moved into the $\mathrm{XOZ}$ plane owing to its symmetrical shape.

\section{Analysis and Discussion}

4.1. Basic Analysis of Spatial Sensitivity. Spatial sensitivity of electrostatic sensor is an important feature for both WSS and OLS, which can reflect the probe ability of inducted charge detection in spatial to eliminate the influence of charge quantity. It is a dimensionless parameter which defined as the negative ratio between probe induced and inducing charge expressed by the following equation:

$$
s(x, z)=-Q(x, z) / q
$$

where $s$ is the electrostatic sensor spatial sensitivity, $Q$ is the probe induced charge, $q$ is the inducing charge, $x$ and $z$ are the position of the inducing charge in coordinate system. The charge value $Q$ can be collected for any point inducing charge by using the above mathematical models. For WSS, it is equivalent to $Q_{\mathrm{WS}}$ in equation (1) with $q, x$ and $z$ are used by $q_{\mathrm{wS}}, x_{\mathrm{wS}}$ and $z_{\mathrm{WS}}$, respectively. Similarly, for OLS, it is equivalent to $Q_{\mathrm{OL}}$ in equation (2) with $q, x$ and $z$ are used by $q_{\mathrm{OL}}, x_{\mathrm{OL}}$ and $z_{\mathrm{OL}}$, respectively. Moreover, considering their sensor structure and installed position, $z_{\mathrm{WS}}$ has values greater than 0 in the axial direction, while $x_{\mathrm{OL}}$ is limited in $(-\mathrm{R}, \mathrm{R})$ at radial direction.

Based on the mathematical model in Section 2.2 above, the electrostatic spatial sensitivity can be simulated through equation (1) and (3) for WSS, while it can be simulated through equation (2) and (3) for OLS. The typical 3D distribution and 2D color map of spatial sensitivity is simulated for both WSS (set $D_{\mathrm{WS}}=10 \mathrm{~mm}$ ) and OLS (set $H_{\mathrm{OL}}=20 \mathrm{~mm}$ and $D_{\mathrm{OL}}=20 \mathrm{~mm}$ ) with the results presented in Figures 4 and 5. From the two figures, the spatial sensitivity of electrostatic WSS and OLS have very different trends in spatial with the changes at the two directions. However, both have symmetry as expected corresponding to their structure as shown in Figure 3. For WSS, the spatial sensitivity is symmetric only at the line $x_{\mathrm{wS}}=0$, while for OLS, it is symmetric at the point of $x_{\mathrm{OL}}=0$ and $z_{\mathrm{OL}}=0$ in two directions. To simplify the process of further investigations, only positive values of $x$ and $z$, which can be regarded as phase-I, are presented for both WSSs with a range of $D_{\mathrm{WS}}$ values (as shown in Figure 6) and OLSs with a range of $H_{\mathrm{OL}}$ and $D_{\mathrm{OL}}$ values (as shown in Figure 7). Any other phases can be inferred through spatial symmetry and all the lengths used in the following parts are millimetre $(\mathrm{mm})$.

Figure 6(a) is a detailed result of WSS in phase-I, which has the same sensor parameter $D_{\mathrm{WS}}=10$ as in Figure 4(a), with 


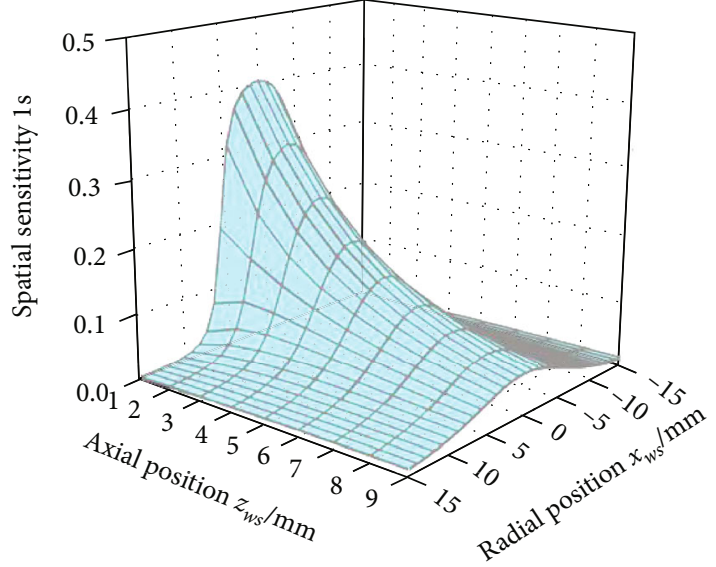

(a) 3D distribution of WSS

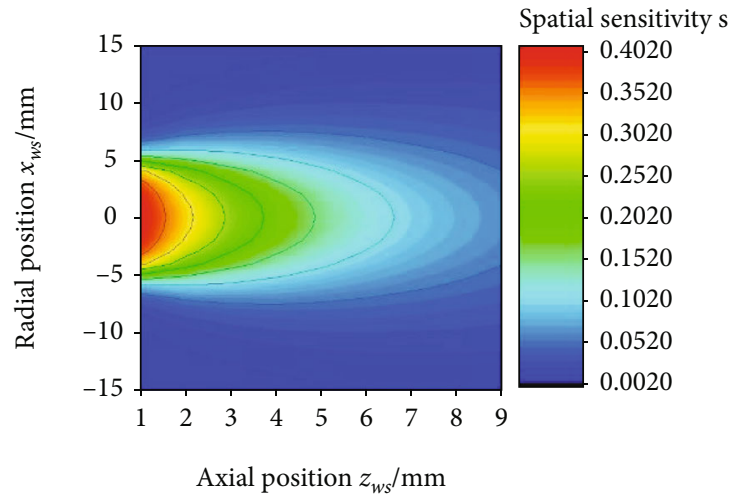

(b) 2D Color map of WSS

FIgURE 4: Simulated spatial sensitivity for an electrostatic WSS with $D_{\mathrm{WS}}=10$ : (a) 3D distribution. (b) 2D Color map.

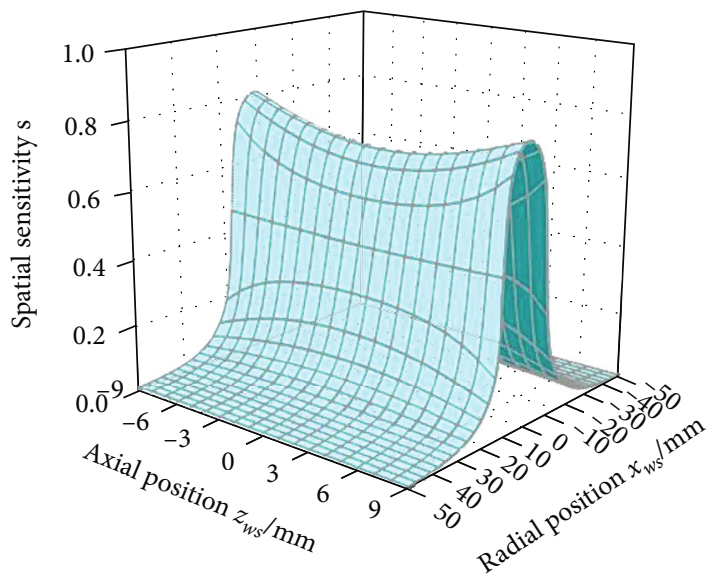

(a) 3D distribution of OLS

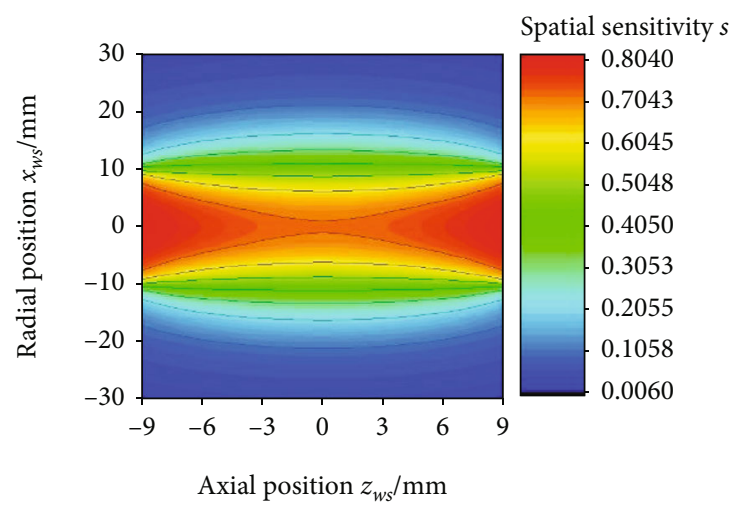

(b) 2D Color map of OLS

FIGURE 5: Simulated spatial sensitivity for an electrostatic OLS with $H_{\mathrm{OL}}=20$ and $D_{\mathrm{OL}}=20$.

more simulated data. Figures 6(b) and 6(c) are the results with half and double of the probe diameter, i.e. $D_{\mathrm{WS}}=5$ and $D_{\mathrm{WS}}=20$. From Figure 6 , it can be seen that the changes of probe diameter have impact on spatial sensitivity for WSS, however their distributions are similar; From Figure 6(b), it can be seen that when the radial coordinate $x_{\mathrm{WS}}=0$, as the axial coordinate increases from 0 to 10 , the value of the $s$ decreases from 0.402 to 0.015 ; when the radial coordinate $x_{\mathrm{ws}}=7$, as the axial coordinate increases from 0 to 10 , the value of sensitivity s first increased from 0.077 to 0.093 and then continued to decrease to 0.015 . Based on the above analysis, we can get the value of $s$ decreases with the increases of axial coordinate inside probe (i.e. $x_{\mathrm{WS}}<R_{\mathrm{WS}}$ ); while the value of $s$ with the increases of axial coordinate outside probe (i.e. $x_{\mathrm{WS}}>R_{\mathrm{WS}}$ ) fist increases and then decreases. It can also be seen that along the increase of radial coordinate $x_{\mathrm{WS}}$ or axial position $z_{\mathrm{ws}}$, i.e. far away from the electrostatic probe, the value of spatial sensitivity $s$ gradually decreases to zero; the closer to the center point of probe $\left(x_{\mathrm{WS}}=0\right.$ and $\left.z_{\mathrm{WS}}=0\right)$, the higher spatial sensitivity $\mathrm{s}$ it has. To any certain position in spatial with the same coordinate of
$x_{\mathrm{WS}}$ and $z_{\mathrm{WS}}$, a higher spatial sensitivity it can get with a larger probe diameter of WSS applied, especially for the area inside the probe.

Figure 7(a) is also a detailed result of OLS in phase-I, which has the same sensor parameter $H_{\mathrm{OL}}=20$ and $D_{\mathrm{OL}}=20$ as in Figure 5(a), with more simulated data. Figures 7(b) and 7(c) are the results with the same 10 value minus and plus of the ring probe length in axial direction, i.e. $H_{\mathrm{OL}}=10$ and $H_{\mathrm{OL}}=30$, without any change of probe diameter $\left(D_{\mathrm{OL}}=20\right)$ in radial direction. Similarly, Figures $7(\mathrm{~d})$ and $7(\mathrm{e})$ are the results with the same 10 value minus and plus of the ring probe diameter in radial direction, i.e. $D_{\mathrm{OL}}=10$ and $D_{\mathrm{OL}}=30$, without any change of probe length $\left(H_{\mathrm{OL}}=20\right)$ in axial direction. Then the results with the same 10 value minus and plus synchronous on both probe length and diameter are also presented as shown in Figure $7(\mathrm{f})$ (i.e. $H_{\mathrm{OL}}=10$ and $D_{\mathrm{OL}}=10$ ) and Figure $7(\mathrm{~g})$ (i.e. $H_{\mathrm{OL}}=30$ and $\left.D_{\mathrm{OL}}=30\right)$.

From Figure 7, it can be seen that the changes of two probe parameters, i.e. length and diameter, influence the characteristics for OLS, however their distributions are 


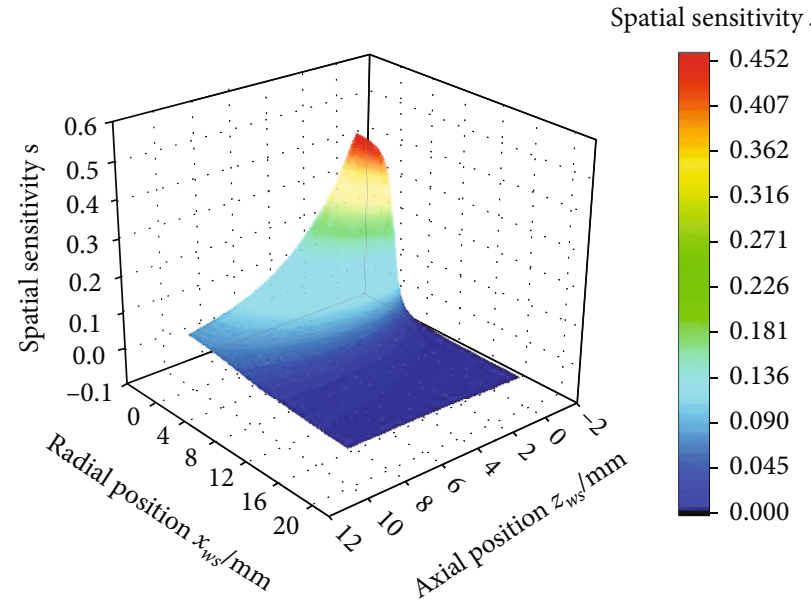

(a) $D_{\mathrm{WS}}=10$

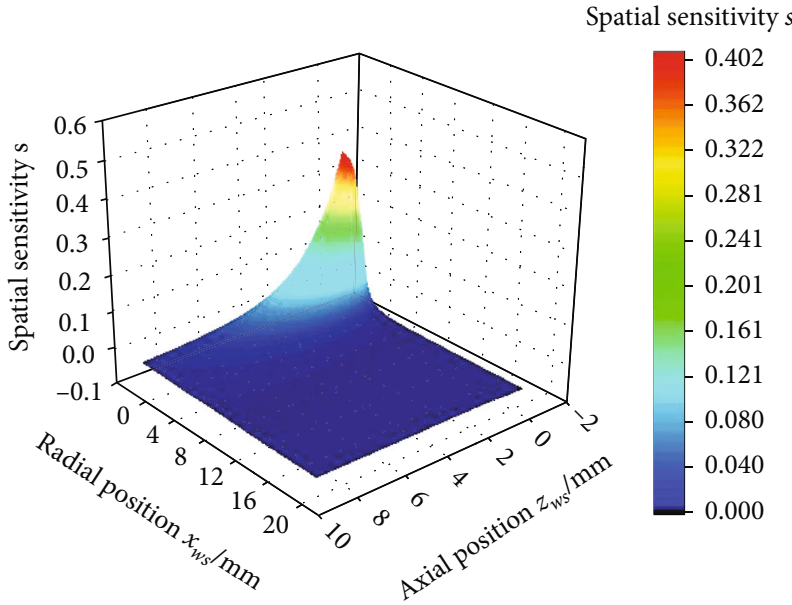

(b) $D_{\mathrm{WS}}=5$

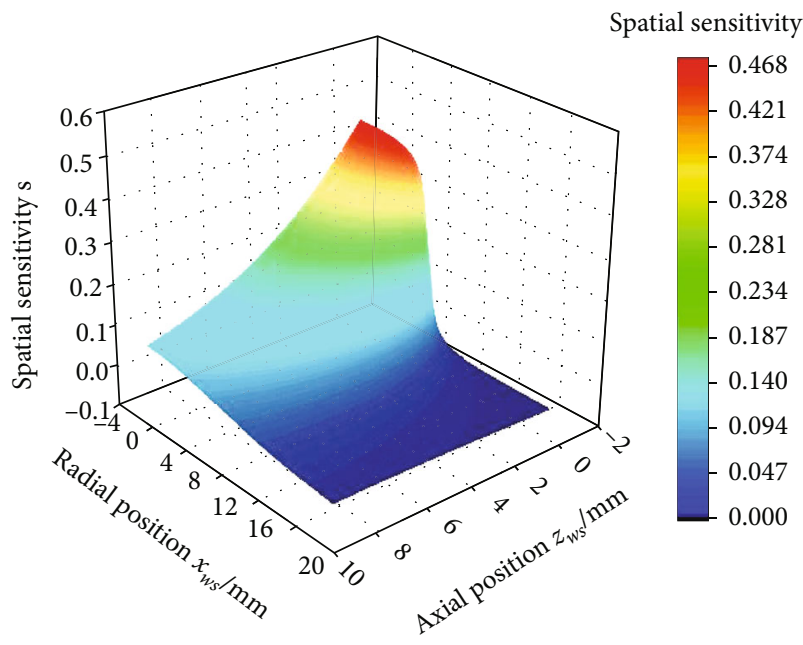

(c) $D_{\mathrm{WS}}=20$

FIgURE 6: Simulated 3D distribution of spatial sensitivity at phase-I for WSSs with different sensor parameters.

similar. The value of $s$ increases with its decrease of axial position $z_{\mathrm{OL}}$ and gets maximum when $z_{\mathrm{OL}}=0$. From Figure $7(\mathrm{a})$, it can be seen that When the axial position $z_{\mathrm{OL}}=5$, the radial position increases from 0 to 10 , and the value of $s$ increases from 0.652 to 0.791 ; when the axial position $z_{\mathrm{OL}}=25$, the radial position increases from 0 to 10 , and the value of $s$ increases from 0.064 Reduced to 0.043 ; this shows $s$ increases with its increase of radial position $x_{\mathrm{OL}}$ inside probe (i.e. $z_{\mathrm{OL}}<L_{\mathrm{OL}}$ ) and decreases outside (i.e. $z_{\mathrm{OL}}>L_{\mathrm{OL}}$ ). For any OLS with a certain probe length and diameter, it can also be seen that with the increase of $z_{\mathrm{OL}}$, i.e. far away from the sensor probe, the spatial sensitivity $s$ value gradually decreases to zero; the closer to the center position of probe $\left(x_{\mathrm{OL}}=L_{\mathrm{OL}}\right.$ and $\left.z_{\mathrm{OL}}=0\right)$, the higher spatial sensitivity s it has. Combined with the results of WSS above, both the trends are in accordance with the electrostatic sensing principle, i.e. the nearer the inducing charge to the surface of probe, the more charge will be induced on the surface with much more effective electric flux.

From Figure 7, it can also be seen that changes of the probe parameters $H_{\mathrm{OL}}$ and $D_{\mathrm{OL}}$ have different influences for spatial sensitivity, respectively, more complexed than WSS in Figure 6. The spatial sensitivity value especially its maximum increases with the increase of $H_{\mathrm{OL}}$ according to Figures 7 (a)7 (c); while it decreases with its increase of DOL inside and increases outside its probe according to Figures $7(\mathrm{a}), 7(\mathrm{~d})$ and $7(\mathrm{e})$. It indicates the OLS with its ring shape probe can to be manufactured with long (higher probe length) and thin (lower probe diameter) to increase its detection accuracy with higher sensitivity inside and lower outside. Therefore, the change trend for spatial sensitivity to specific spatial position become complex with the increase or decrease of both probe length and diameter, meanwhile also seen from Figures 7 (a), $7(\mathrm{f})$ and $7(\mathrm{~g})$. Just simply enlarge or reduce the size of probe is not suitable for OLS, based on the view of spatial sensitivity.

4.2. Further Analysis of Other Essential Characteristics. Apart from spatial sensitivity, there are some other characteristics, e.g. probe ratio, static sensitivity and efficiency, for both electrostatic WSS and OLS. According to their definitions, each formula is listed in Table 2. 


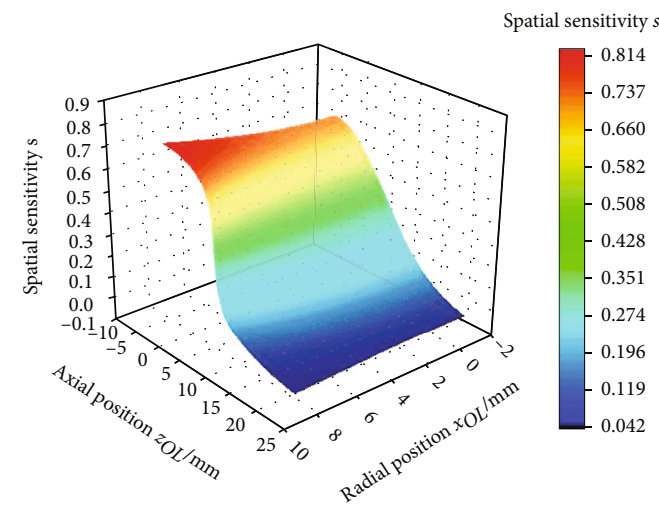

(a) $H_{\mathrm{OL}}=20$ and $D_{\mathrm{OL}}=20$

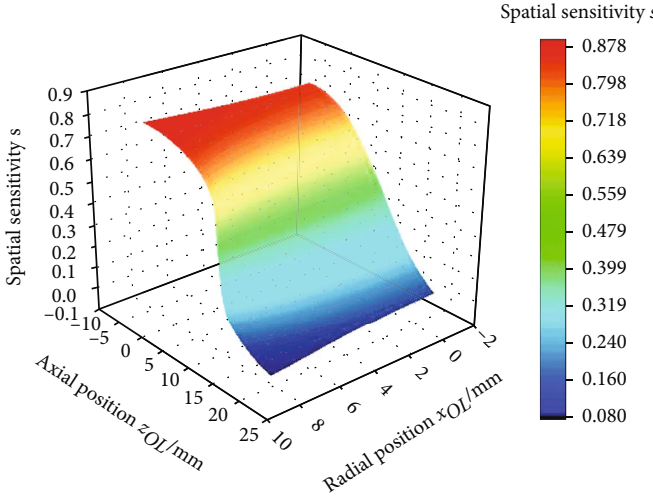

(c) $H_{\mathrm{OL}}=30$ and $D_{\mathrm{OL}}=20$

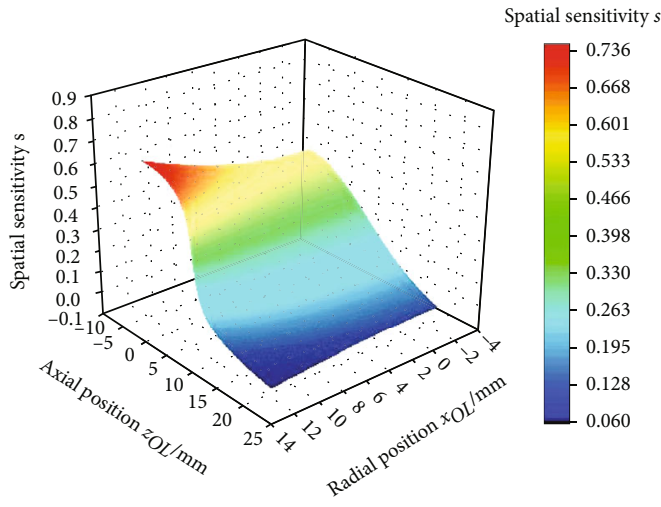

(e) $H_{\mathrm{OL}}=20$ and $D_{\mathrm{OL}}=30$

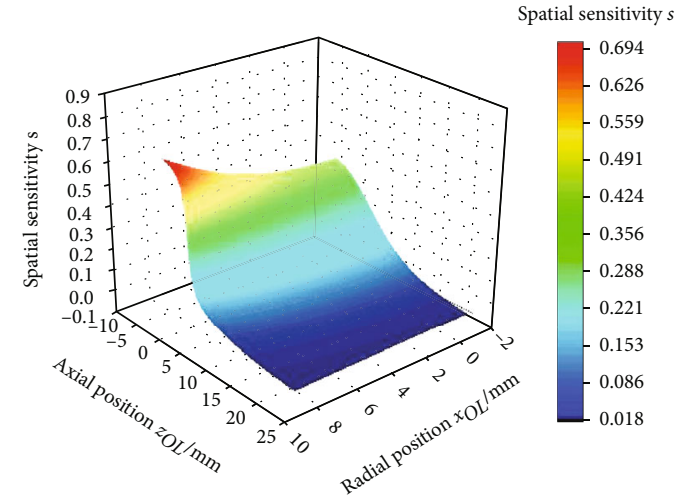

(b) $H_{\mathrm{OL}}=10$ and $D_{\mathrm{OL}}=30$

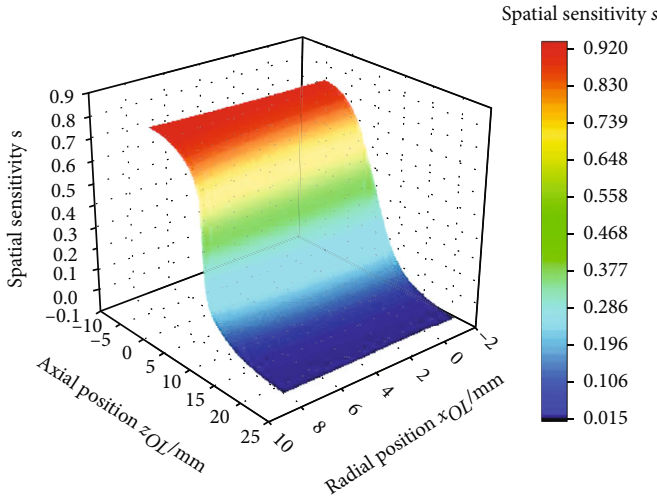

(d) $H_{\mathrm{OL}}=20$ and $D_{\mathrm{OL}}=10$

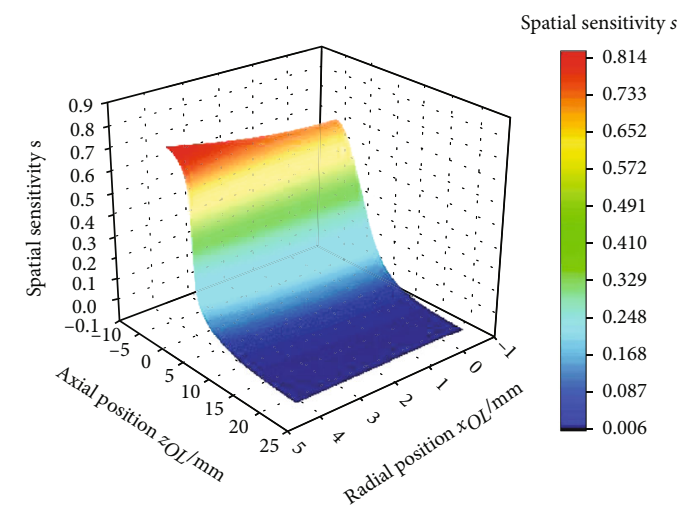

(f) $H_{\mathrm{OL}}=10$ and $D_{\mathrm{OL}}=10$

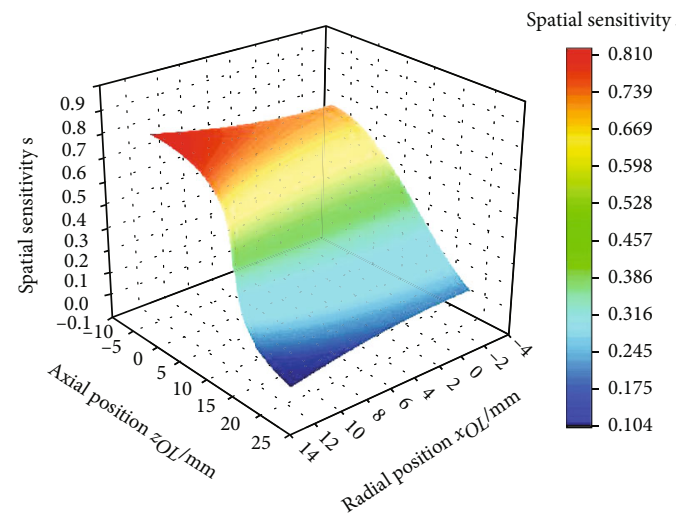

(g) $H_{\mathrm{OL}}=30$ and $D_{\mathrm{OL}}=30$

FIGURE 7: Simulated 3D distribution of spatial sensitivity at phase-I for OLSs with different sensor parameters. 
TABLE 2: Characteristics comparison of electrostatic WSS and OLS.

\begin{tabular}{lcc}
\hline Electrostatic sensor & WSS & Equations \\
Characteristics & $R_{\mathrm{WS}} / Z_{\mathrm{WS}}$ & OLS \\
\hline Probe ratio $\lambda$ & $-\left(\mathrm{Q}_{\mathrm{WS}}\left(0, z_{\mathrm{WS}}\right) / q_{\mathrm{WS}}\right) \times 100 \%$ & $H_{\mathrm{OL}} / D_{\mathrm{OL}}$ or $L_{\mathrm{OL}} / R_{\mathrm{OL}}$ \\
Theoretical efficiency $\eta$ & $\left(\mathrm{Q}_{\mathrm{WS}}^{\prime}\left(0, z_{\mathrm{WS}}\right) / q_{\mathrm{WS}}^{\prime}\right) \times 100 \%$ & $-\left(Q_{\mathrm{OL}}(0,0) / q_{\mathrm{OL}}\right) \times 100 \%$ \\
Real efficiency $\eta^{\prime}$ & $\Delta U_{\mathrm{WS}}^{\prime}\left(0, z_{\mathrm{WS}}\right) / \Delta q_{\mathrm{WS}}^{\prime}$ & $\left(Q_{\mathrm{OL}}^{\prime}(0,0) / q_{\mathrm{OL}}^{\prime}\right) \times 100 \%$ \\
Static sensitivity $s^{\prime}$ & & $\Delta U_{\mathrm{OL}}^{\prime}\left(x_{\mathrm{OL}}, 0\right) / \Delta q_{\mathrm{OL}}^{\prime}$ \\
\hline
\end{tabular}

where $q_{\mathrm{WS}}^{\prime}$ and $q_{\mathrm{OL}}^{\prime}$ are the charge of sensor input for WSS and OLS, respectively, in the experiment; $Q_{\mathrm{WS}}^{\prime}$ and $Q_{\mathrm{OL}}^{\prime}$ are the transformed maximum value of charge of sensor output for WSS and OLS, respectively, in the experiment; $\Delta q_{\mathrm{WS}}^{\prime}$ and $\Delta q_{\mathrm{OL}}^{\prime}$ are the increment of sensor input charge for WSS and OLS, respectively, in the experiment; $\Delta U_{\mathrm{WS}}^{\prime}$ and $\Delta U_{\mathrm{OL}}^{\prime}$ are the increment of sensor outsput voltage for WSS and OLS, respectively, in the experiment.

Probe ratio $\lambda$ is a physical parameter based on probe structure for electrostatic sensors. To OLS, it is simply to calculate and equal to ratio of sensor probe length $H_{\mathrm{OL}}$ and diameter $D_{\mathrm{OL}}$ (or ratio of half probe length $L_{\mathrm{OL}}$ and radius $R_{\mathrm{OL}}$ ), which indicates when an OLS is manufactured, the parameter is confirmed without any possible changes. The concept is extended to WSS with an added center-distance $\mathrm{Z}_{\mathrm{WS}}$, considering there is only one structure parameter, i.e. diameter. The parameter $\lambda$ for WSS is the ratio of sensor probe radius $R_{\mathrm{Ws}}$ and probe center distance $\mathrm{Z}_{\mathrm{WS}}$, which means the value would be determined both by the sensor probe and installation position. Thus, different from OLS, the ratio is various to a manufactured WSS before corresponding to a specific applied position. Because the value of $H_{\mathrm{OL}}, D_{\mathrm{OL}}, R_{\mathrm{WS}}$ and $Z_{\mathrm{WS}}$ are all positive and larger than $0, \lambda$ also has values in the range of $(0,+\infty)$ theoretically. The parameter probe ratio $\lambda$ is essential in the electrostatic sensor efficiency, another characteristic which is also investigated in the following.

Electrostatic sensor efficiency is a significant parameter that reflects the sensor's detection ability, which has a close relevant to spatial sensitivity and probe ratio. It has two characteristics, i.e. theoretical efficiency $\eta$ (based on mathematical simulation) and real efficiency $\eta^{\prime}$ (based on experimental outputs). The theoretical efficiency forms a reference curve to indicate the sensor's detection ability, as shown in Figure 8, while the real efficiency is a range of given values tested from the experiments corresponding to a certain manufactured sensor. The value of theoretical efficiency equals to the spatial sensitivity multiply by $100 \%$ at a special position (geometrical center for OLS and axial direction for WSS) with variable of probe ratio. It is based on the theory of simulation results that the spatial at the special position sensitivity is fixed under the same ratio $\lambda$ values despite its variation in sensor length or diameter.

To theoretical efficiency, from Figure 8, it can be seen that the two curves have similar trend, although the structure of the two electrostatic sensors are different. The value of theoretical efficiency increases with probe ratio for both

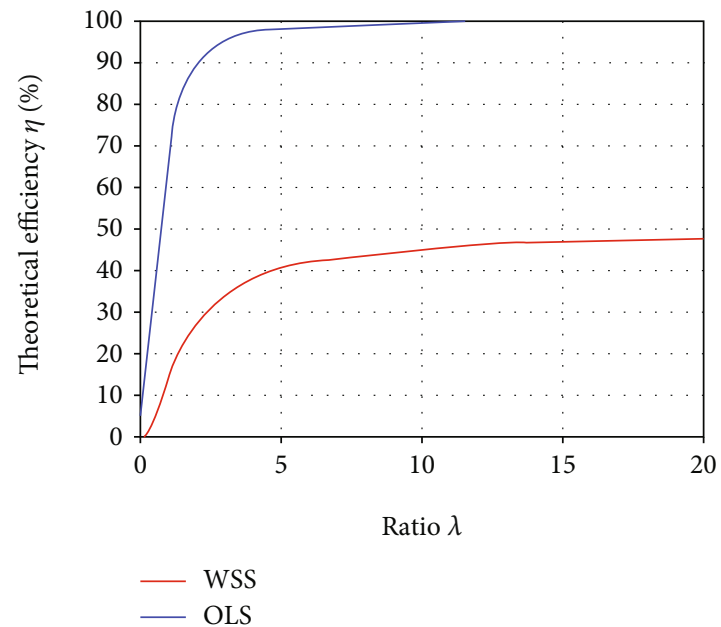

FIgURE 8: Theoretical efficiency of WSS and OLS.

WSS and OLS; They both increase rapidly at the beginning, especially in the range $\lambda<5$, and then became much slower to the end; At one side with $\lambda$ approaches 0 , the value of theoretical efficiency for both WSS and OLS are both very close to 0 ; while at another side with a quite large value for $\lambda$, the value of the theoretical efficiency are very close to $50 \%$ and $100 \%$ for WSS and OLS, respectively.

This result is in accordance with the electrostatic charging principle with more electric flux terminates and more charges induced on surface to increase the efficiency of electrostatic sensors. To OLS theoretically when the ratio approaches 0 , i.e. the probe diameter is far larger than the probe length, few electric fluxes can terminate on probe surface and its efficiency value also approaches 0 ; while as its ratio close to infinity, i.e. sensor probe length is far larger than probe diameter, all flux can terminate on probe surface then its efficiency value approaches $100 \%$. To WSS theoretically, as the ratio approaches 0 , i.e. the center-distance is far larger than the probe-radius, similarly few electric fluxes can terminate on probe surface and its efficiency value also approaches 0 ; while as the ratio approaches infinity, i.e. the probe-radius is far larger than the center-distance, all the electric flux at only one side (facing the probe) will terminate on the probe surface and its efficiency value approaches $50 \%$. Each manufactured electrostatic sensor follows this result theoretically for WSS and OLS, respectively, as a reference theoretical efficiency curve. 


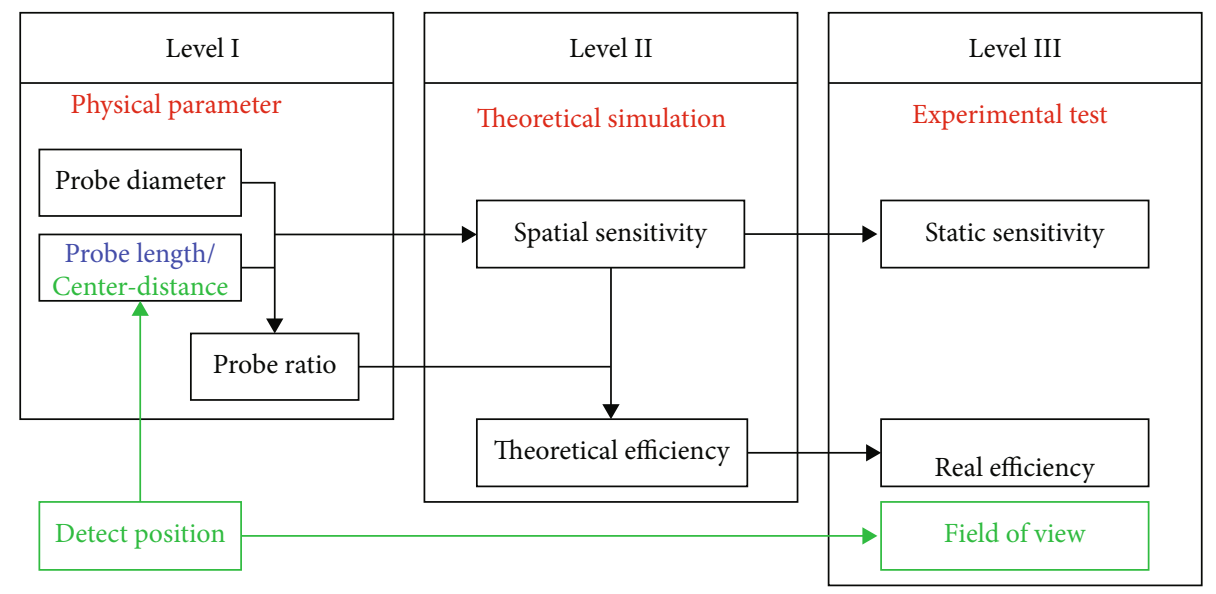

Figure 9: A unified three-level system of electrostatic WSS and OLS.

Corresponding to the basis of electrostatic spatial sensitivity and sensor theoretical efficiency, the two more characteristics, i.e. static sensitivity and real efficiency, are both obtained and calculated from sensor input and output through experiment by using the same setup of calibration system. The system has oil charging and charge measurement components with electrostatic sensor installation and signal acquisition devices [27]. Due to the fact that it is hard to give the accurate position of the inducing charge at a fixed moment and the electrostatic sensor real output is voltage in experiment, the static sensitivity indicates the ratio of voltage increment detected from sensor output to the charge increment collected sensor input at a series of fixed special coordinate, seen from Table 2. To WSS, the effective position is axial direction within a detectable range with radial direction $x_{\mathrm{Ws}}=0$; while to OLS, it is at radial direction within the probe radius with axial position $z_{\mathrm{OL}}=0$. According to experiments, the results can be plotted by a well fitted polynomial fit over experimental data along with a mathematical expression as the static sensitivity of the tested electrostatic sensor. The real efficiency indicates the ratio between detected output to collected input at the fixed coordinate with $100 \%$ multiplied as well, also seen from Table 2. To WSS, the specific position is also at axial direction within a detectable range with radial direction $x_{\mathrm{WS}}=0$; while to OLS, it is at the geometrical center with the coordinate $(0,0)$. The values of Q' can be calculated and regarded as the noted Qmax by transforming the electrostatic output signal, corresponding to the position according to experiments. The specific procedures and steps of experiment and calculation for static sensitivity and real efficiency with manufactured electrostatic sensors in the experimental setup, along with tested results, which has been described in detail in previous investigations $[26,27]$, are omitted here.

The detectable range in WSS mentioned above indicates another characteristic which is introduced and named 'field of view' in previous investigations. It indicates the electrostatic sensor maximum measurable range at space when the inducing charge shows in front of the sensor probe. The undetected part is the "blind area" of electrostatic sensor. The tested results with the same experimental setup are also presented in $[26,27]$. Although the characteristic 'field of view' is investigated and involved in both WSS and OLS, after a comparison in actual application, it is found that the characteristic is more useful for WSS than OLS. The value of this characteristic is an essential reference for WSS as the detective distance in installation (within in the range of the sensor probe to the detection point) to ensure the electrostatic monitoring effectiveness. While for OLS as illustrated in section 2, therefore it is positioned in the oil pipe, all the charged debris in the oil line would pass through the region of the sensor probe theoretically without any need of the characteristic field of view.

4.3. Comprehensive Analysis of Electrostatic Sensor. So far, with the analysis of all the characteristics investigated above on electrostatic WSS and OLS, a unified three-level system is further extracted and presented to classify parameters and characteristics for electrostatic sensors, as shown in Figure 9. Both WSS and OLS follow this strategy and system mostly with the same characteristics in black in this figure. The special and unique parts are also marked in green (WSS only) and blue (OLS only). All the characteristics are divided into three levels, i.e. physical parameter as level I, theoretical simulation as level II and experimental test as level III. It means level I is related to the physical structure and parameter of electrostatic sensor, while level II and level III is based on the theoretical simulation and experimental test, respectively. These characteristics have inner relationship which exist both in the same level and between different levels.

In level I, it includes the probe diameter, probe length (OLS only) or center-distance (WSS only), and their combination probe ratio. These physical parameters are the most basic characteristics for electrostatic sensor which can be easily measured and calculated on probe. They also determine the basic structure of the electrostatic sensor.

In level II, it includes the electrostatic spatial sensitivity and their theoretical efficiency as characteristics of electrostatic sensor on simulation. The spatial sensitivity is a distribution in space based on the mathematical model and calculated with the set of the two probe size parameters. With the results 
of spatial sensitivity and parameter probe ratio, the theoretical efficiency is extracted and presented with a fixed curve for all WSSs and OLSs, respectively, while the value of spatial sensitivity changes with different probe sizes.

In level III, it includes the static sensitivity and real efficiency corresponding to the former spatial sensitivity and their theoretical efficiency, along with the unique parameter field of view for WSS only. All these characteristics in this level are based on the results of experimental tests on a setup of electrostatic calibration system. Each manufactured electrostatic sensor with different probe has its own values on these characteristics.

All the characteristics above in three levels need to be carefully considered for electrostatic sensors before their application in actual industry to ensure the effectiveness and reliability on condition morning. It is also worth mention that the diagram for electrostatic sensors in Figure 9 is a summary of current useful characteristics based on previous investigations. It may be expanded with more significant characteristics founded or extracted and added in with further researches, considering the development of electrostatic monitoring technique.

\section{Conclusions}

Sensor performance is essential in the process of electrostatic monitoring of tribo-contacts. However, the two typical types, i.e. WSS and OLS, have very different structures with the same sensing principle. It is important to investigate their parameters and characteristics with a contrast analysis and unify them in a system for electrostatic monitoring technique. This paper analysed their differences and similarities based on their mathematical model and theoretical simulation. The main conclusions are summarized:

(1) Owing to their different sensor structures, the distributions of spatial sensitivity to the probe are quite different for electrostatic WSS and OLS. However, the distributions for a series of among one type sensor (WSSs or OLSs only) with different probe parameters are similar. In addition, they all have symmetry according to the values of $3 \mathrm{D}$ simulation. They are symmetric at Z axis for WSSs and at the point of geometric centre for OLSs, which are in accordance with their sensor structure

(2) For the simplified distribution of spatial sensitivity in phase-I, the value for WSS increases at both axial and radial direction inside the probe range; while the value for OLS decreases with its axial position and increases with its radial position inside probe. It also exists outside however the values are much smaller than those inside

(3) Although WSS and OLS distributions of spatial sensitivity are different, the trends of sensor theoretical efficiency related to the parameter probe ratio for both WSS and OLS are similar. The value of theoretical efficiency increases, rapidly at first and then slowly towards to a certain value (50\% for WSS and $100 \%$ for OLS), with the increase of probe ratio, which agrees with the electrostatic sensor charging principle

A unified three level system is extracted and presented for electrostatic sensor parameters and characteristics based on physical structure, theoretical simulation, and experimental test, along with their inner correlation.

\section{Data Availability}

The data used to support the findings of this study are included within the article.

\section{Conflicts of Interest}

The authors declare no conflicts of interest.

\section{Acknowledgments}

This research was supported by National Natural Science Foundation of China, grant number 51705221, 91860139凶 52072176 and Jiangsu Government Scholarship for Overseas Studies. The authors would like to thank Prof. Michael G. Pecht for his support to the leading author's study at University of Maryland, College Park.

\section{References}

[1] S. Cheng, M. H. Azarian, and M. G. Pecht, "Sensor systems for prognostics and health management," Sensors, vol. 10, no. 6, pp. 5774-5797, 2010.

[2] B. Wang, Y. Han, D. Tian, and T. Guan, "Sensor-based environmental perception Technology for Intelligent Vehicles," Journal of Sensors, vol. 2021, Article ID 8199361, 14 pages, 2021.

[3] W. Mao, J. He, J. Tang, and Y. Li, "Predicting remaining useful life of rolling bearings based on deep feature representation and long short-term memory neural network," Advances in Mechanical Engineering, vol. 10, no. 12, p. 18, 2018.

[4] L. Xin, Z. Hongfu, C. Jing, S. Jianzhong, L. Ruochen, and $\mathrm{X}$. Yutong, "Real-time electrostatic monitoring of Wear debris for wind turbine gearbox," Transaction of Nanjing University of Aeronautics \& Astronautics, vol. 34, no. 2, pp. 195-204, 2017.

[5] Y. Zhang, A. Wang, and H. Zuo, "Roller bearing performance degradation assessment based on fusion of multiple features of electrostatic sensors," Sensors, vol. 19, no. 4, p. 824, 2019.

[6] Y. Li, C. Wang, and C. Yao, "Multi-sensors image fusion via NSCT and GoogLeNet," Transactions of Nanjing University of Aeronautics \& Astronautics, vol. 37, no. S, pp. 88-94, 2020.

[7] K. Zolna, P. B. Dao, W. J. Staszewski, and T. Barszcz, "Nonlinear cointegration approach for condition monitoring of wind turbines," Mathematical Problems in Engineering, vol. 2015, 11 pages, 2015.

[8] B. I. A. N. Yixiang, Z. H. A. N. G. Yi, Z. H. A. N. G. Yanjun, G. O. N. G. Junjie, J. I. N. Hong, and D. A. I. Longchao, "Design and fabrication of a bio-inspired Accleration sensor using liquid mass blocks," Transactions of Nanjing University of Aeronautics \& Astronautics, vol. 36, no. 1, pp. 160-167, 2019. 
[9] L. Wang, R. J. K. Wood, T. J. Harvey, and H. E. G. Powrie, "Electrostatic charge-a novel condition monitoring technology," Condition Monitor, vol. 248, pp. 5-9, 2007.

[10] Z. Wen, J. Hou, and J. Atkin, "A review of electrostatic monitoring technology: the state of the art and future research directions," Progress in Aerospace Sciences, vol. 94, pp. 1-11, 2017.

[11] O. D. Tasbaz, R. J. K. Wood, M. Browne, H. E. G. Powrie, and G. Denuault, "Electrostatic monitoring of oil lubricated sliding point contacts for early detection of scuffing," Wear, vol. 230, no. 1, pp. 86-97, 1999.

[12] S. Morris, Real-Time Electrostatic Charge Monitoring of the Wear Surfaces and Debris Generated by Sliding Bearing Steel Contacts, University of Southampton, 2003.

[13] J. E. Booth, K. D. Nelson, T. J. Harvey et al., "The feasibility of using electrostatic monitoring to identify diesel lubricant additives and soot contamination interactions by factorial analysis," Tribology International, vol. 39, no. 12, pp. 1564-1575, 2006.

[14] R. Liu, H. Zuo, J. Sun, and L. Wang, "Electrostatic monitoring of wind turbine gearbox on oil-lubricated system," Proceedings of the Institution of Mechanical Engineers, Part C: Journal of Mechanical Engineering Science, vol. 231, no. 19, pp. 36493664, 2017.

[15] H. E. G. Powrie and C. E. Fisher, "Engine health monitoring: towards total prognostics," in IEEE Aerospace Applications Conference Proceedings, vol. 3, pp. 11-20, Snowmass, CO, USA, March 1999.

[16] Z. Wen, H. Zuo, and M. G. Pecht, "Electrostatic monitoring of gas path debris for aero-engines," IEEE Transactions on Reliability, vol. 60, no. 1, pp. 33-40, 2011.

[17] L. Pengpeng, Z. Hongfu, and S. Jianzhong, "The electrostatic sensor applied to the online monitoring experiments of combustor carbon deposition fault in aero-engine," IEEE Sensors Journal, vol. 14, no. 3, pp. 686-694, 2013.

[18] S. Morris, R. J. K. Wood, T. J. Harvey, and H. E. G. Powrie, "Electrostatic charge monitoring of unlubricated sliding wear of a bearing steel,"Wear, vol. 255, no. 1-6, pp. 430-443, 2003.

[19] T. J. Harvey, R. J. K. Wood, and H. E. G. Powrie, "Lectrostatic wear monitoring of rolling element bearings," Wear, vol. 263, no. 7-12, pp. 1492-1501, 2007.

[20] J. E. Booth, T. J. Harvey, R. J. K. Wood, and H. E. G. Powrie, "Scuffing detection of TU3 cam-follower contacts by electrostatic charge condition monitoring," Tribology International, vol. 43, no. 1-2, pp. 113-128, 2010.

[21] T. J. Harvey, R. J. K. Wood, G. Denuault, and H. E. G. Powrie, "Effect of oil quality on electrostatic charge generation and transport," Journal of Electrostatics, vol. 55, no. 1, pp. 1-23, 2002.

[22] T. J. Harvey, R. J. K. Wood, G. Denuault, and H. E. G. Powrie, "Investigation of electrostatic charging mechanisms in oil lubricated tribo-contacts," Tribology International, vol. 35, no. 9, pp. 605-614, 2002.

[23] J. Sun, R. J. K. Wood, L. Wang, I. Care, and H. E. G. Powrie, "Wear monitoring of bearing steel using electrostatic and acoustic emission techniques," Wear, vol. 259, no. 7-12, pp. 1482-1489, 2005.

[24] M. Craig, T. J. Harvey, R. J. K. Wood, K. Masuda, M. Kawabata, and H. E. G. Powrie, "Advanced condition monitoring of tapered roller bearings, part 1," Tribology International, vol. 42, no. 11-12, pp. 1846-1856, 2009.
[25] Y. Zhang, H. Zuo, and F. Bai, "Feature extraction for rolling bearing fault diagnosis by electrostatic monitoring sensors," Proceedings of the Institution of Mechanical Engineers, Part C: Journal of Mechanical Engineering Science, vol. 229, no. 10, pp. 1887-1903, 2015.

[26] H. MAO, H. ZUO, and H. WANG, "Electrostatic Sensor Application for On-Line Monitoring of Wind Turbine Gearboxes," Sensors, vol. 18, no. 10, p. 3574, 2018.

[27] L. Ruochen and Z. Hongfu, "Mathematical model, simulation, and experimental calibration of electrostatic wear-site sensor," IEEE Sensors Journal, vol. 17, no. 8, pp. 2428-2438, 2017.

[28] R. C. Liu, H. F. Zuo, J. Z. Sun, and L. Wang, "Simulation of electrostatic oil line sensing and validation using experimental results," Tribology International, vol. 105, pp. 15-26, 2017.

[29] H. Mao, H. Zuo, H. Wang, Y. Yin, and X. Li, "Debris recognition methods in the lubrication system with electrostatic sensors," Mathematical Problems in Engineering, vol. 2018, Article ID 8043526, 15 pages, 2018.

[30] Y. Xu, Q. Zheng, J. Geng et al., "Synergistic effects of electroless piston ring coatings and nano-additives in oil on the friction and wear of a piston ring/cylinder liner pair," Wear, vol. 422-423, pp. 201-211, 2019.

[31] Z. Luo, J. Yu, Y. Xu et al., "Surface characterization of steel/ steel contact lubricated by PAO6 with novel black phosphorus nanocomposites," Friction, vol. 9, no. 4, pp. 723-733, 2021.

[32] Y. Xu, Q. Zheng, R. Abuflaha et al., "Influence of dimple shape on tribofilm formation and tribological properties of textured surfaces under full and starved lubrication," Tribology International, vol. 136, pp. 267-275, 2019. 